\title{
Solve VRPPD with Improved Bacteria Optimization Algorithm
}

\author{
LI Bo ${ }^{1,2}$, GUO Chen ${ }^{1, a}$, NING Tao ${ }^{2}$,WEI Yingqi ${ }^{2}$ \\ ${ }^{1}$ College of Information Science and Technology, Dalian Maritime University, Dalian, Liaoning \\ Province, China \\ ${ }^{2}$ Institute of Software, Dalian Jiaotong University, Dalian, Liaoning Province, China \\ adaliannt@126.com
}

\begin{abstract}
Keywords: vehicle routing problem with pickup and delivery, improved bacteria foraging optimization algorithm, non dominate sorting

Abstract. In this paper, an improved bacteria foraging optimization algorithm based on different constraint conditions is proposed to solve the vehicle routing problem with pickup and delivery (VRPPD). At first, the mathematical model is established aiming at minimizing the dispatching time and the total cost. Secondly, the paper proposes the method with dynamic variable step factor, as well as propagation threshold and death threshold to copy the excellent individuals and eliminate the inferior individuals. Finally, the improved method is applied to the CMTnX and CMTnY, and its effectiveness is verified from the result of comparison with some existing algorithms.
\end{abstract}

\section{Introduction}

In the VRP with Pickup and Delivery, the heterogeneous vehicle fleet based on multiple terminals must meet a set of transportation requests. Each request is defined by a pickup point and the corresponding delivery point. The objective function(s) is generally minimizing the delivery cost. The previous work on VRPPD was conducted for dial-a-ride scenarios [1]. It was first examined by Wilson and Weissberg [2], and motivated by the demand-responsive transportation systems. A parallel insertion heuristic was proposed by Roy et al [3] for the multiple VRPPD in the context of the transportation of disabled persons. Since a fair amount of requests are known in advance, these are used by means of time-spatial proximity criteria to create initial routes for all vehicles starting at the beginning of the day. Madsen, Ravn, and Rygaard [4] implemented a generalized version of this approach for a partly dynamic dial-a-ride problem. Their algorithm can minimize the waiting time for the vehicle as well as the break time. Local search for the VRPPD was first considered by Psarafits [5], who extended the ideas of Lin and Kernighan. A decade later, Bent R and Hentenryck presented another local search heuristic for the VRPPD [6]. The algorithm involves two phases, both using arc exchange procedures. In the construction phase, it tries to find an initial feasible route allowing infeasibility and penalizing the violation of restrictions in the objective function [7]. There are a variety of practical applications about VRPPD, including the transport of the disabled and elderly, sealift and airlift of cargo, as well as the pickup and delivery for overnight carriers. Perspectives on this growing field were offered by Solomon and Desrosiers, et al [8].

An improved bacterial foraging optimization algorithm (IBFOA) based on different constraint condition is proposed in this paper. The effectiveness is verified through the application to the CMTnX and CMTnY. Here, the above efforts can be extended by reviewing important recent developments and offering our view for future directions.

\section{Model of VRPPD}

Identify request by two nodes of $i$ and $n+i$, respectively, correspond to the pickup and delivery. It is possible for different nodes to represent the same geographical location. Next, denote the set of pickup nodes by $P=\{1, \ldots, n\}$ and the set of delivery nodes by $D=\{n+1, \ldots, 2 n\}$. Further, define $N=P \cup D$. If request $i$ consists of transporting $d_{i}$ units from $i$ to $n+i$, let $l_{i}=d_{i}$ and $l_{n}+i=-d_{i}$.

$K$ represents the set of vehicles. Because not all the vehicles can serve all request points, each 
vehicle $k$ has a specific set $N_{k}=P_{k} \cup D_{k}$ associated with it, where $N_{k}, P_{k}$, and $D_{k}$ are appropriate subsets of $N, P$ and $D$ respectively. For each vehicle $k$, the network is defined as $G_{k}=\left(V_{k}, A_{k}\right)$. $V_{k}=N_{k} \bigcup\{o(k), d(k)\}$ represents the set of nodes inclusive of the origin, $o(k)$, and destination, $d(k)$, depots for vehicle $k$, respectively. The subset $A_{k}$ of $V_{k} \times V_{k}$ comprises all feasible arcs. The capacity of vehicle $k$ is given by $C_{k}$, and its travel time and cost between distinct nodes $i, j \in v_{k}$, by $t_{i j k}$ and $c_{i j k}$, respectively.

The mathematical model of VRPPD may be described as follows:

$$
\begin{aligned}
& \min Z=\sum_{k=1}^{K} \sum_{j=1}^{N} f_{k} x_{i j k}+\sum_{k=1}^{K} \sum_{i=1}^{N} \sum_{j=1}^{N} p_{k} d_{i j} x_{i j k} \\
& y_{i j k}=\left\{\begin{array}{l}
1 \\
0
\end{array} \forall i, j=1,2, \ldots, N ; \quad \forall k=1,2, \ldots, k ;\right. \\
& y_{i k}=\left\{\begin{array}{l}
1 \\
0
\end{array} \quad \forall i=1,2, \ldots, N ; \quad \forall k=1,2, \ldots, k ;\right. \\
& \sum_{k=1}^{K} \sum_{i=1}^{N} x_{i j k}=1, \forall j=1,2, \ldots, N \\
& \sum_{k=1}^{K} \sum_{i=1}^{N} x_{i j k}-\sum_{k=1}^{K} \sum_{i=1}^{N} x_{j i k}=0 \\
& 0 \leq U_{i k}-\sum_{j=1}^{N} X_{i j k}\left(q_{i}-p_{i}\right) \leq Q \\
& 0 \leq \sum_{j=1}^{M} q_{j}\left\{\sum_{i=1}^{N} X_{i j k}\right\} \leq r \cdot Q \\
& \sum_{i=1}^{N} v_{i} y_{i k} \leq V_{k}, k=1,2, \ldots, K \\
& P \leq \frac{\sum_{i=1}^{N} p_{i} \times y_{i k}}{\sum_{i=1}^{N} q_{i} \times y_{i k}} \leq 1
\end{aligned}
$$

The significance of symbol in the mathematical model is as follows:

$x \quad$ a path;

$y \quad$ a path state;

$f_{k} \quad$ the fixed cost of vehicle $k$;

$p_{k}$ the travel cost of vehicle $k$ in per $\mathrm{km}$;

$M$ the number of customers (the customer here represents the person who needs to send or receive orders);

$K$ the number of vehicles which are providing the service;

$d_{i j} \quad$ the distance between customer $i$ and customer $j$;

$Q$ the maximum loads of vehicles;

$q_{i} \quad$ the delivery quantity for the customer $i$;

$p_{i} \quad$ the pickup quantity for the customer $i$;

$v \quad$ the ratio between goods volume and the maximum load when the vehicle leaves the logistic center;

$U_{\mathrm{ik}} \quad$ the load of vehicle $\mathrm{k}$ after leaves the customer $i$;

$P \quad$ ratio between pickup and delivery.

Eq (1) represents the objective function including the fixed cost and traveling cost for the vehicle. $\sum_{k=1}^{K} \sum_{j=1}^{N} f_{k} x_{i j k}$ is the total fixed cost and $\sum_{k=1}^{K} \sum_{i=1}^{N} \sum_{j=1}^{N} p_{k} d_{i j} x_{i j k}$ is the total traveling cost. Eq (2) represents the leaving of vehicle $k$ from $i$ to $j$. Eq (3) indicates that vehicle $k$ is serving the customer $i$. Eq (4) indicates that the customer $i$ can be served only by vehicle $k$. Eq (5) indicates that any customer can be served only once at most. Eq (6) indicates the total load of vehicle $k$ can not exceed its capacity 
of $Q$. Eq (7) indicates that the initial loading rate of vehicle $k$ should be less than 1 to reserve some space for pickup. Eq (8) indicates that the total volume of the goods in vehicle $k$ can't exceed the upper limit. Eq (9) indicates that the pickup service should be executed in the completion of a certain amount of delivery service, so as to minimize the cost of cargo handing.

\section{Improved Bacteria Foraging Optimization Algorithm}

The IBFOA based on crowding distance and a variable step size adaptive strategy is proposed here. The improved algorithm is as follows.

Step 1: Initialization. Set the position of $P_{o}$, the population size of $S_{P}$, the chemotatic times of $N_{c}$, the reproductive times of $N_{r}$ and the eliminate times of $N_{d}$.

Step 2: Chemotaxis. Generate the unit vector, making the bacteria individual tumble and swim, the position of the $i^{t h}$ individual is updated according to the following equation [9]:

$$
\begin{aligned}
\theta^{i}(k+1, j, l) & =\theta^{i}(k, j, l)+\operatorname{cs}(i) \varphi(i) \\
\varphi(i) & =\frac{\Delta(i)}{\sqrt{\Delta^{T}(i) \Delta(i)}}
\end{aligned}
$$

In Eq $(10) \theta^{i}(k, j, l)$ is the position of the $i^{\text {th }}$ bacteria individual in the $k^{\text {th }}$ chemotaxis, $j^{\text {th }}$ production and $l^{\text {th }}$ elimination; $c s(i)$ is the chemotatic step size; $\varphi(i)$ is the direction vector of unit length; $\Delta(i)$ is the random vector; $\delta \in(0,1)$ is the crowding distance factor. The adjustable step formula is as follow:

$$
\operatorname{cs}(i)=m f\left[\frac{\delta(\delta+1)}{\delta+\operatorname{crowd}}-\delta\right] B l
$$

In Eq (12), $m_{\mathrm{f}}$ is the step size adjustment factor, crowd is the crowding distance, crowd $=\left(n_{c} / S_{P}\right)$, $n_{c}$ is the number of the partners in the sensing range, $B_{1}$ is the length of the search interval. If crowd is small, the bacteria individual will optimize in larger step size; otherwise, it will be in smaller step size. The above operation makes the algorithm have strong global search ability in the early stage and have strong local search ability in the late stage.

Step 3: Reproduction. The "propagation threshold" and "death threshold" are introduced into the reproduction. Propagation threshold: If the bacteria individual has to reproduce because the absorption of nutrients during the swimming process, then it reaches "propagation threshold". Death threshold: if the bacteria individual does not absorb enough nutrients to survive and be eliminated, then it reaches "death threshold". Reproduce the excellent individual which has reached "propagation threshold" and eliminate the ones which has reached "death threshold". If the reproduction time is equal to the predetermined number, go to step 4; otherwise, go to step 2.

Step 4: Dispersion. If the dispersion time of bacteria colony reaches the predetermined number, the algorithm will stop; otherwise, the bacterial colony will be dispersed to any direction and go to step 2 to rechemotaxis and reproduction.

\section{Analysis and Verification}

In order to verify the efficiency of the proposed method, the standard problems of literature [10] are solved by IBFOA. The comparison with BFO (Bacterial foraging optimization algorithm) [9], QPSO (Quantum-behaved particle swarm optimization) [11] is shown in Table 1. From Table 1, it can be seen that IBFOA can not only obtain more non-dominated solutions but obtain the current optimal solution in instance, while it is the worst with BFO. For example, for case $10 \times 7$, although both QPSO and IBFOA have obtained three non-dominated solutions, the solution $(53,102,52)$ obtained by QPSO is dominated by both $(53,101,52)$ and $(52,102,51)$ obtained by IBFOA, the solution $(53,101,53)$ obtained by QPSO is dominated by $(52,102,51)$ obtained by IBFOA, the solution $(53,101,53)$ obtained by QPSO is dominated by both $(53,101,52)$ and $(52,101,53)$ obtained by IBFOA. The above can indicate that IBFOA is more effective than the existing algorithms. $D_{\mathrm{j}}$ is the deliver loading and $P_{\mathrm{j}}$ is the request loading at the point $j$. 
In Table $1, S_{n}(\mathrm{n}=1,2,3,4)$ is the different solution; $T_{t}$ is the total time of certain orders; $C_{t}$ is the total cost; $L_{a}$ is the average loading rate of single vehicle.

Table 1. Results of four algorithms and CPLEX

\begin{tabular}{ccccccccccc}
\hline \multirow{2}{*}{$D_{j} \times P_{j}$} & objective & \multicolumn{2}{c}{ BFO } & \multicolumn{1}{c}{ QPSO } & \multicolumn{5}{c}{ IBFOA } \\
\cline { 3 - 11 } & value & $S_{1}$ & $S_{2}$ & $S_{1}$ & $S_{2}$ & $S_{3}$ & $S_{1}$ & $S_{2}$ & $S_{3}$ & $S_{4}$ \\
\hline $5 \times 5$ & $T_{t}$ & 52 & & 52 & 53 & 54 & 52 & 52 & 53 & 52 \\
& $C_{t}$ & 72 & & 72 & 72 & 73 & 72 & 71 & 72 & 72 \\
& $L_{a}$ & 50 & & 51 & 49 & 48 & 50 & 49 & 49 & 49 \\
\multirow{5}{*}{$10 \times 5$} & $T_{t}$ & 56 & 56 & 55 & 56 & 55 & 55 & 56 & 55 & 55 \\
& $C_{t}$ & 117 & 116 & 117 & 116 & 114 & 116 & 116 & 114 & 115 \\
& $L_{a}$ & 53 & 53 & 53 & 53 & 54 & 53 & 53 & 53 & 52 \\
$15 \times 10$ & $T_{t}$ & & & 53 & 52 & 53 & 53 & 52 & 52 & \\
& $C_{t}$ & & & 102 & 103 & 101 & 101 & 102 & 101 & \\
& $L_{a}$ & & & 52 & 52 & 53 & 52 & 51 & 53 & \\
& $T_{t}$ & 48 & & 48 & 47 & 48 & 48 & 47 & 48 & 47 \\
& $C_{t}$ & 43 & & 82 & 83 & 82 & 82 & 83 & 82 & 82 \\
& $L_{a}$ & 47 & & 47 & 46 & 46 & 47 & 46 & 46 & 47 \\
\hline
\end{tabular}

\section{Conclusion}

A multi-objective VRPPD mathematical model according to different constraints is established in this paper firstly. Considering the characteristics of the dynamic variable step factor, the improved bacteria foraging optimization algorithm is proposed to solve VRPPD. Finally, the simulation results of CTMnX demonstrate that the proposed method can improve the efficiency of iterative searching and obtain more non-dominated solutions than the existing algorithms. All the above can verify the effectiveness of the proposed method. The future study direction should lie in considering the cloud algorithm.

\section{Acknowledgment}

This work is partially supported by the National Natural Science Foundation of China (No.51579024, No.61374114), Liaoning Provincial Social Science Planning Foundation of China (No. L16BGL008) and Dalian Social Science Planning Foundation of China (No. 2016dlskyb104) .

\section{References}

[1] Desaulniers, Guy, Jacques Desrosiers, Andreas Erdmann, Marius M. Solomon, and François Soumis. "VRP with Pickup and Delivery", The Vehicle Routing Problem, 2002.

[2] H. Wilson and H. Weissberg. Advanced dial-a-ride algorithms research project: Final report. Technical Report R76-20, Department of Civil Engineering, MIT, Cambridge, MA, 1976.

[3] S. Roy, J-m. Rousseau, G. Lapalme, and J.A. Ferland. Routing and scheduling for the transportation of disabled persons-the algorithm. Technical Report TP 5596E, Centre de Recherche sur les Transports, Montreal, Canada, 1994.

[4] O.B.G. Madsen, H.F. Ravn, and J.M. Rygaard. A heuristic algorithm for a dial-a-ride problem with time windows, multiple capacities, and multiple objectives. Annals of Operations Research, vol.60, pp.193-208, 2005.

[5] H.N. Psaraftis. k-Interchange procedures for local search in a precedence-constrained routing problem. European Journal of Operational Research, vol.13, pp.391-402, 2003.

[6] Tao NiNG, Chen Guo, Rong Chen. A Novel Method for Dynamic Vehicle Routing Problem. The Open Cybernetics \& systemic journal, 2015, 9:1-5.

[7] NING Tao, Huang Ming, Liang Xu, et al. A novel dynamic scheduling strategy for solving 
flexible job-shop problems, Journal of Ambient Intelligence \& Humanized Computing, 25(7): 721-729, 2016.

[8] CUI Jingjing, SUN Yanming, CHENG Lanxiu. Improved bacteria foraging optimization algorithm for Job-Shop scheduling problems [J]. Application Research of Computers, 2011, 28(9):3324-3326.

[9] LIU Qin. Differential Evolution Bacteria Foraging Optimization Algorithm for Bus Scheduling Problem[J]. Journal of Transportation Systems Engineering and Information Technology, 2012,12(2):156-161.

[10] Mitra S. A parallel clustering technique for the vehicle routing problem with split deliveries and pickups. Journal of the Operational Research Society, 2008, 59(11): 1532-1546.

[11] Sun J, Fang W, Palade V, et al. Quantum-behaved particle swarm optimization with Gaussian distributed local attractor point. Applied Mathematics and Computation, 2011, 218(7): 3763-3775. 